\title{
LOCAL QUASIOPTICAL RESONATOR DIAGNOSTICS OF SEMICONDUCTOR WAFERS
}

\author{
Igor Dorofeeva , Grigorii Dunaevskii, Alexander Badin, Kirill Dorozhkin, Vitaly Bessonov, \\ Sergey Khodovitskiy
}

National Research Tomsk State University, 634050, Tomsk, Russia

\begin{abstract}
The method of measurement of reflection coefficient behind an orifice in one of reflectors of the quasioptical open resonator is considered. According to the applied mathematical model, the method has the restriction which is consist in use of a short-wave approximation for the orifice size (it dimension is much more than a wavelength). For evaluation of limits of applicability of this method the experimental setup in the threecentimetric range of wavelengths consist of the quasioptical open resonator and the system of positioning of the measuring probe is created. By the experimental study of distribution of fields behind an orifice in the reflector of the open resonator it is found that the method, except restriction of mathematical model, has also restrictions, due to the wave's interference, reflected from edges of this orifice. It causes a deviation from the uniform amplitude distribution of the field in an opening that can lead to increase in an error of a reflection coefficient measuring. It is also shown that by decrease of the sizes of an orifice formation of a bunch with small divergence, but with significantly more expressed distribution of the field localized about a bunch axis in an aperture is possible. It can be used for diagnostics of inhomogeneity of samples with higher locality.
\end{abstract}

\section{Introduction}

Now intensive development of element base of millimetric range of wavelengths on the basis of semiconductor technologies is observed. At the same time especially significant demands are placed on a prime material of which scheme elements are made. For millimetric and submillimetric (terahertz) ranges it is possible to single out from perspective materials $\mathrm{GaN}$ and materials on its basis [1], in particular, applied to creation of HEMT transistors [2, 3]. For successful realization of final devices the great value gets an opportunity of preliminary diagnostics of wafers with high space resolution.

It is essential that such diagnostics can and has to be carried out at those frequencies at which future semiconductor elements will be used. Millimeter waves to fall into to the range in which it is effectually to use quasi-optics elements instead of some traditional waveguide and resonator elements. Some of quasioptical approaches were realized also in the centimeter range therefore it is the most reasonably to expand the range of their use, having used already tried-and-true techniques. On more low frequencies for a research of

\footnotetext{
a Corresponding author:idorofeev@mail.tsu.ru
} 
plane-parallel samples which size in transversal directions significantly exceeds a wavelength usually use a method of the free space which analog in quasioptics is the measurements in a lens waveguide. However the sensitivity of such methods is small. Significantly resonator techniques have much higher sensitivity. The quasioptical resonator is the open resonator which is often called the Fabri-Perot resonator. At the same time intra resonator methods of diagnostics of sheets have the locality limited to beam aperture and are intended only for materials with low losses [4].

\section{The method of measurements}

In [5] the quasioptical resonator technique of measurement of local values of the module and a phase of a reflection coefficient from plane-parallel samples through an orifice in one of reflectors of the open resonator is presented. In this case the locality of measurement can be almost 10 times more, than in case of intra resonator location of sample. Besides, there is an opportunity to investigate value distributions of a reflection coefficient on the surface of material and also to work with samples which size does not allow carrying out measurements in the free space. The developed method was successfully applied to a research the plane-layered materials with significant losses.

It should be noted that for this method the locality is limited to the orifice size which in turn, determines beam divergence outside of the resonator. Two contradictory requirements are imposed to the size of an orifice. On the one hand the size of the orifice has to be larger in comparison with a wavelength to provide the flat phase front and small divergence of a wave behind it, on the other side of its size has to be small in comparison with diameter of a beam on a mirror of the open resonator if to consider inhomogeneity of the field in the directions, perpendicular resonator axes. In practice it is rather difficult to meet these two requirements therefore often it is necessary to be limited to compromise option. Within the mathematical model realized for a method it is impossible to carry out any estimates of border of influence of the sizes of an orifice therefore it is reasonable to carry out the experimental studies of correctness of the theoretical assumptions.

First of all it is necessary to estimate distribution of the field behind an orifice. The experimental setup on the basis of the open resonator was created for this purpose, in one of reflectors of which the orifice for interaction of the field of resonance oscillations with the studied object was located. Considering scalability of the methods of diagnostics with change of a wavelength based on use of open resonators, researches can be carried out in the most convenient sites of the frequency range. The three-centimeter range of wavelengths was chosen for the purpose of simplification of the device of scanning of the measuring probe and increases its resolution capability.

\section{Experimental setup}

The open resonator consisted of the flat and concave mirrors (Figure 1). Its geometrical sizes corresponded to a quasioptical case, that is there were much more wavelengths and made: diameter of a plane mirror is $30 \mathrm{~cm}$, concave mirror is $40 \mathrm{~cm}$ and distance between them $53 \mathrm{~cm}$. Radius of curvature of a concave mirror $-120 \mathrm{~cm}$. 


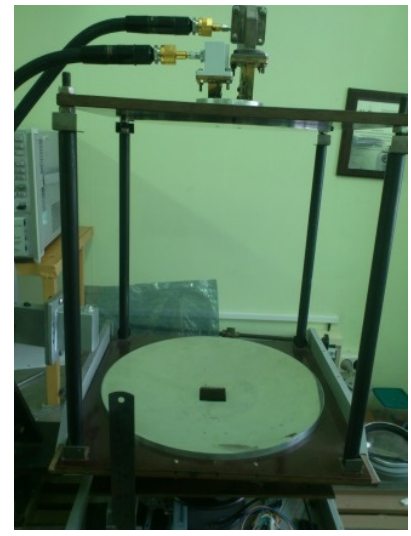

Fig. 1. Open resonator for measurements.

In the center of a concave mirror the orifice of square shape with the side of $38 \mathrm{~mm}$ was located. On a plane mirror elements of communication of the open resonator with external circuits (Figure 2) was created. These elements represented the pieces of waveguides converging on a narrow wall to narrow (about 100 microns) splits.

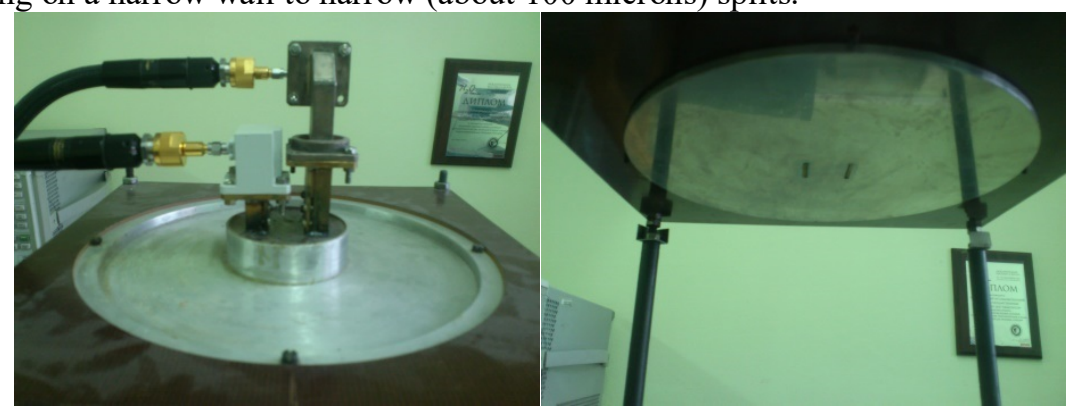

Fig. 2. Couple elements of open resonator with external circuits.

Oscillations with a vector of an electric field which was orthogonal to splits were excited in the open resonator. Thereby polarization degeneration was removed. Two couple elements were used so that there was an opportunity to define value of a transmission coefficient via the open resonator. The sides of the orifice on the concave mirror were located strictly in parallel and perpendicular to splits.

Below a concave mirror the table for samples which had a possibility of movement in the direction of an axis of the open resonator (Figure 3) was located. The plane of a table is made from polished brass and served also for calibration (the reflection coefficient module from it was accepted to unit, a phase -- for $180^{\circ}$ ).

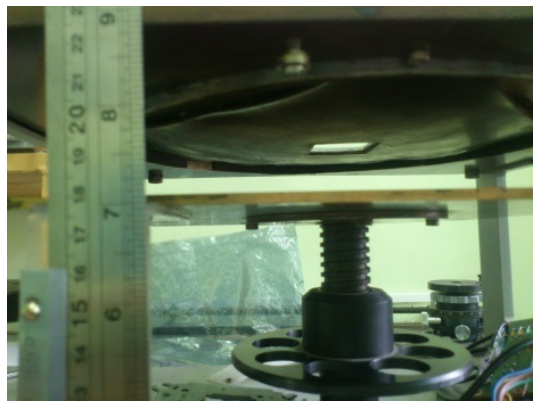

Fig. 3. Table for calibration and samples with the movement mechanism. 
The surface of the concave mirror was covered with the absorber for decrease of influence of the resonances resulting from formation of standing waves between a sample and a mirror.

For measurement of field's distributions with a probe method it is necessary to choose probes which sizes there is much less wavelength, at the same time it is better to use the absorbing probes than scattering. However in this case there is no need to use the pointwise probe. As the orifice is square, that electric field to one of its sides will be orthogonal, relative others - in parallel. As the tangential component of the field on the relevant sides will tend to zero, deviations from the uniform distribution will be much more in the direction, perpendicular to the direction of a vector of an electric field. Therefore the long threadlike probe from the thin absorbing carbon fiber was used (Figure 4).

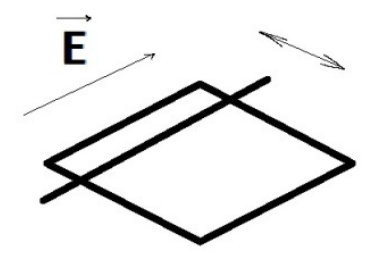

Fig.4. Probe location relative to electrical field vector.

The probe was fixed on a frame and moved in the direction, perpendicular to a vector of an electric field by means of the mechanism given by the step engine with a step of 158 microns. At each position of the probe the value of a transmission coefficient of the open resonator on a resonant frequency of the corresponding mode which change was in proportion to the power absorbed by the probe was fixed. Its value can be estimated as follows $P_{p}=\frac{1}{2} \sigma \int_{-a}^{a} E^{2} d l$. Here $\sigma$ - conductivity of material of the absorbing thread. Integration is carried out along the probe. As the field along it is orthogonal to the sides of an orifice, it can be considered the homogeneous in this direction in case the sizes of an orifice are rather big in comparison with a wavelength. So $P_{p}=a \sigma E^{2}$. Thus, change of a transmission coefficient of the open resonator on a resonant frequency is proportional to the value of an electric field square in an orifice. The probe moved to the planes, the most close to a mirror taking into account its thickness and thickness of the absorber.

\section{The results of measurements}

The network analyzer Agilent E8363B was used to measurements of values of transmission coefficient of the open resonator on a resonant frequency. At measurements in a frequency band which size was determined by the pieces of waveguides forming coupling elements 8 main fundamental modes of the open resonator were used. Observed dates of distribution of three of them are given in graphs on Figure 5. Not to encumber graphs the most reference dependences were chosen. The type of the others is similar to given. 


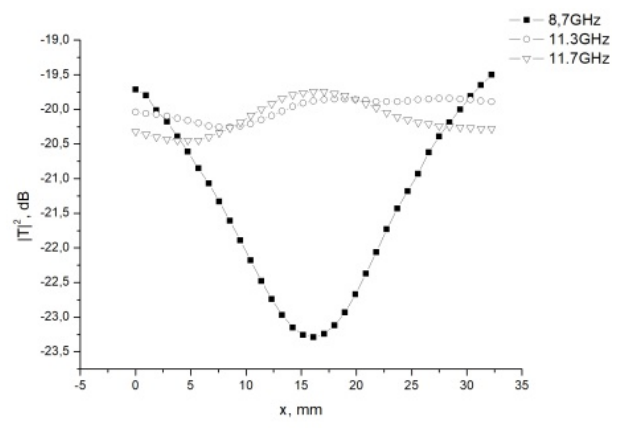

Fig.5. Field distribution near the measurement orifice in the direction, perpendicular to electrical vector.

As appears from the received results, most the uniform distribution of the field was observed for mode with a resonant frequency of $11.3 \mathrm{GHz}$, but not predictably, on the most high frequency. For it the field has oscillations. The interference of the fields scattering by the opposite sides of a square orifice is the reason of it. For the most low-frequency mode it is possible to observe rather non-uniform distribution similar created by quasioptical beam. Thus, the assumption which is that this orifice cuts out from a resonance beam the site with the uniform amplitude distribution of the field is not carried out not only for orifice insufficiently larger in comparison with a wavelength, but also and at rather larger. However it is possible to achieve enough homogeneous distributions of the field practically on all area of an orifice by selection of it size.

Deviations of distribution of the field from the uniform will lead to increase of an error of definition of a reflection coefficient from the sample corresponding to value of a reflection coefficient for a plane wave. Therefore it is necessary to use an orifice of the variable sizes for measurements in a wide frequency band.

However in some cases, for problems of diagnostics of uniformity of exemplars an absolute value cannot matter, resolution capability is much more important. Its high value will able to find out inhomogeneity of more low scale. And in this case, as appears from Figure 5, decrease of the sizes of an orifice leads to increase in locality of interaction of the electromagnetic beam created by a measuring orifice and a sample.

For evaluation of influence of such non-uniformity of distribution of the field to beam divergence behind an orifice, dependences of transmission coefficient of the open resonator on the location of a measuring table were measured. These dependences are given in Figure 6.

If to take for criterion of divergence of a beam the value of decrease of a maximum of a transmission coefficient at increase in distance between a table and a mirror, then, apparently from these graphs, these values are quite comparable among themselves. 

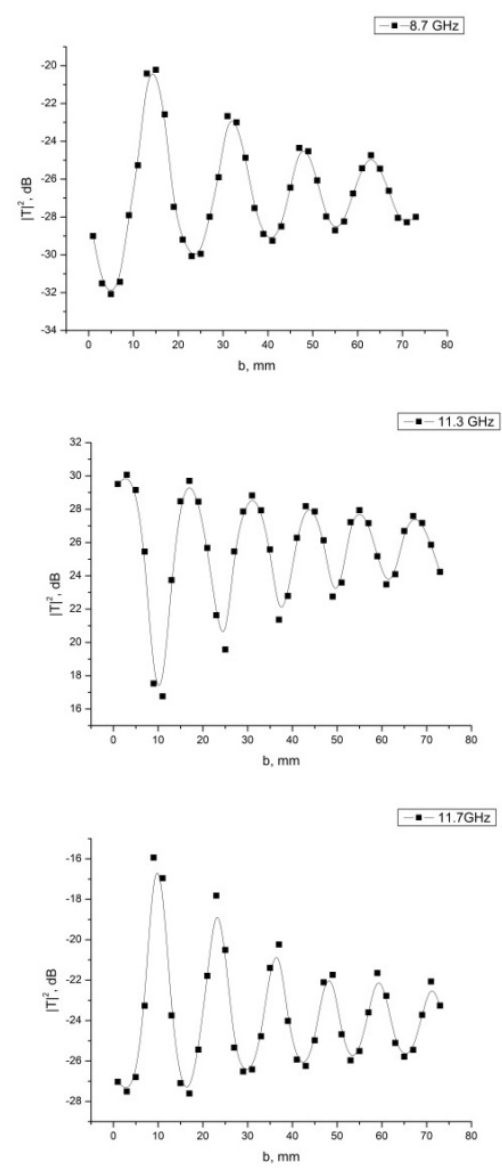

Fig.6. The dependence of transmission coefficient of open resonator on resonant frequency of corresponding mode from the distance between the mirror and the table.

\section{Conclusion}

Thus, as a result of the experimental study of the field distribution it is established that the method of measurement of a reflectivity behind a measuring orifice in the reflector of the open quasioptical resonator has restrictions not only a short-wave approximation (the size of an orifice have to be big in comparison with a wavelength), but also long-wave because of an interference of the fields reflected from edges of an orifice.

If this method to use for diagnostics of the relative values of inhomogeneity of samples, by means of a small orifice it is possible to create a beam which has a small divergence, but higher locality of the field.

Use of the open resonator rather just allows expanding a method in a high part of a gigahertz frequency range and also in terahertz. The method does not demand exemplars of the larger sizes, works in the wide range of change of their parameters, provides high locality, provides a possibility of scanning of samples. In the presence of a source of electromagnetic oscillations it can be realized as the self-contained unit. 


\section{Acknowledgements}

This research was conducted as part of the project № 14.578.21.0240 (RFMEFI57817X240) Ministry of Education and Science of the Russian Federation.

\section{References}

[1] K. Ahi, Opt. Eng. 56, 1. (2017)

[2] T. Otsuji, M. Shur, IEEE Microwave Magazine 15, 43. (2014)

[3] W. Knap, D. Coquillat, N. Dyakonova, F. Teppe, O. Klimenko, H. Videlier, S. Nadar, J. Łusakowski, G. Valusis, F. Schuster, B. Giffard, T. Skotnicki, C. Gaquière, A. El Fatimy, Comptes Rendus Physique,11, 433. (2010)

[4] B. Yang, S. Katz, K. Willis, M. Weber, I. Knezevic, S. Hagness, J. Booske, IEEE Trans. on Terahertz Science and Techn. 2, 449. (2012)

[5] G. Dunaevskii, An Open Quasioptical Transformers. (Izdatelstvo NTL, Tomsk, 2006) 